\title{
Time Domain Channel Model for the THz Band
}

\author{
Kazuhiro Tsujimura, Kenta Umebayashi*, Joonas Kokkoniemi ${ }^{\dagger}$, and Janne Lehtomäki ${ }^{\dagger}$ \\ * Tokyo University of Agriculture and Technology, Koganeishi, Tokyo \\ $\ddagger$ Centre for Wireless Communications (CWC), Oulu, Finland
}

\begin{abstract}
Impulse response is proposed for wireless nanosensor networks which is used the terahertz band (THz band: 0.1-10 THz) and short range (1-100 cm). There is not only a line-ofsight $(\mathrm{LoS})$ path but also a reflected path in nanosensor networks. In $\mathrm{THz}$ band, rough surface on the reflector significantly effect due to very short wavelength. This paper focuses on frequency domain and time domain channel models and, for wireless communication analysis the impulse response is very important specially. Frequency domain channel model represents molecular absorption and rough surface effect which are unique in $\mathbf{T H z}$ band. And time domain channel model shows delayed wave even in LoS path. Reflected path has significantly strong effect to received signal from $\operatorname{LoS}$ path at long distance between transmitter and receiver than at short distance, relatively. These channel model leads to development of $\mathrm{THz}$ band communication technique.
\end{abstract}

Index Terms-Causality, channel models, impulse response, molecular absorption, reflected path, terahertz band

\section{INTRODUCTION}

Envisioned applications for nanodevices include health monitoring, plants monitoring, haptic interfaces, interconnected offices, damage detection systems, and ultra-highspeed on-chip communication [1], [2]. For a nanodevice to be capable of reporting sensing information or receiving tasks, communication between devices is required [1]. Electromagnetic (EM) communication and molecular communication have been proposed to enable device-to-device communication in wireless nanosensor networks (WNSNs) [1].

One of the suggested frequency bands for nanodevices is the terahertz band $(0.1-10 \mathrm{THz})$ [3], which is our frequency of interest. In contrast to the UHF band, the effect of molecular absorption needs to be considered in the THz band [3]. Molecular absorption and its impact on transmittance of the channel were studied in [3]. Transmittance is the ratio of received signal power to transmitted signal power in the channel for frequency domain and is modeled with the Beer-Lambert law. Then, the molecular absorption loss in the $\mathrm{THz}$ channel is derived by the Beer-Lambert law [3]. Molecular absorption is decided by the composition of a medium, relative humidity (RH), pressure, and temperature, significantly. The signal in $\mathrm{THz}$ band experiences frequency selective fading.

Time domain channel model is come from the transmittance in [4]-[6]. [4] took into account of molecular absorption and antenna response and they proposed Time Spread OnOff Keying (TS-OOK) for both of single and multi user. [5] focused on not only molecular absorption but also a particle scattering which comes from aerosols such as water droplet, and their link was assumed as a line-of-sight (LoS). Our

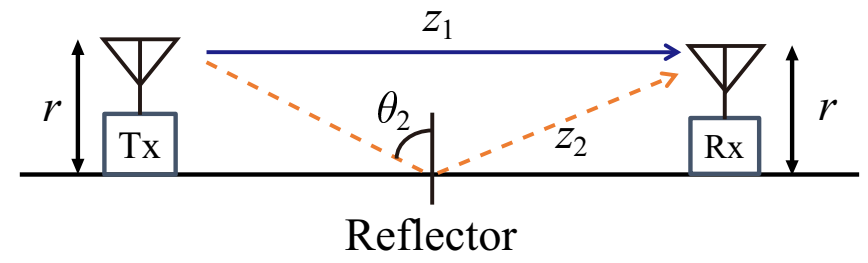

Fig. 1. Multipath scenario $(M=2)$ with $\operatorname{LoS}$ and reflected path. (C)2019 IEEE. Reprinted, with permission, from IEEE Transaction on Terahertz Science and Technology.

previous research in [6] focused on impulse response as a time domain channel model by considering causality.

In this paper, at first we show a derivation of an impulse response as the channel model by a transmittance information. Next, several example of time impulse responses are shown.

\section{FREQUENCY DOMAIN CHANNEL MODEL FOR WNSN}

There may be multiple paths in WNSN, such as LoS path and reflected paths in [7]. So we assume two path model in which one path is LoS and the other path is reflected path like in Fig. 1. In this multipath, the parameters are as follows: $n_{i}=1, r=1 \mathrm{~cm}$, and $z_{1}=1-100 \mathrm{~cm}$. Spreading loss and molecular absorption are considered in the LoS path in [4]. The reflected path should be taken into account roughness of reflector surface. For a simple channel model between a transmitter and a receiver, antenna characteristics are not considered in this paper. The frequency response of the multipath channel $H(f)$ is defined by

$$
H(f)=\sum_{m=1}^{M} H_{m}\left(f, z_{m}\right),
$$

where $f, z_{m}$, and $H_{m}\left(f, z_{m}\right)$ are the frequency, the distance of the $m$ th path, and the frequency response of the $m$ th path, respectively. The frequency response $H_{1}\left(f, z_{1}\right)=H_{1, \operatorname{los}}\left(f, z_{1}\right)$ corresponds to the LoS path and $H_{m}\left(f, z_{m}\right)=H_{m, \text { refl }}\left(f, z_{m}\right)$ for $m \neq 1$ corresponds to the $m$ th reflected path.

\section{A. LoS path model}

The distance between the transmitter and the receiver is set to $z_{1} \mathrm{~cm}$. The transmittance $\left|H_{1}\left(f, z_{1}\right)\right|^{2}$ is defined by

$$
\left|H_{1}\left(f, z_{1}\right)\right|^{2}=\frac{P\left(f, z_{1}\right)}{P(f, z=0)},
$$

where $f$ is the frequency, $P(f, z=0)$ represents the transmitted signal power, $P\left(f, z_{1}\right)$ is the received signal power and 
$H_{1}\left(f, z_{1}\right)$ is the frequency response. The path loss is given by the transmittance as [3]

$$
\text { Path loss }[\mathrm{dB}]=-10 \log _{10}\left(\left|H_{1}\left(f, z_{1}\right)\right|^{2}\right) \text {. }
$$

As can be seen in (2), the transmittance contains the amplitude component of the frequency response, but not the phase component. By considering the spreading loss and the molecular absorption loss, the transmittance (2) can be calculated as [3]

$$
\left|H_{1}\left(f, z_{1}\right)\right|^{2}=\left[A_{\text {abs }}\left(f, z_{1}\right) \times A_{\text {spread }}\left(z_{1}\right)\right]^{-1},
$$

where $A_{\text {spread }}\left(z_{1}\right)$ is the spreading loss and $A_{a b s}\left(f, z_{1}\right)$ is the molecular absorption loss. The spreading loss in a LoS link for an ideal isotropic transmitter is $A_{\text {spread }}\left(z_{1}\right)=4 \pi z_{1}^{2}$. The molecular absorption loss $A_{a b s}\left(f, z_{1}\right)$ can be described by the line absorption loss $A_{l a}\left(f, z_{1}\right)$ and the continuum absorption loss $A_{c a}\left(f, z_{1}\right)$ as [8]

$$
A_{a b s}\left(f, z_{1}\right)=A_{l a}\left(f, z_{1}\right) \times A_{c a}\left(f, z_{1}\right) .
$$

with $A_{l a}\left(f, z_{1}\right)$ and $A_{c a}\left(f, z_{1}\right)$ given by

$$
\begin{aligned}
& A_{l a}\left(f, z_{1}\right)=\exp \left(\sum_{i} k_{l a}^{i}(f) z_{1}\right) \\
& A_{c a}\left(f, z_{1}\right)=\exp \left(\sum_{j} k_{c a}^{j}(f) z_{1}\right),
\end{aligned}
$$

where $i$ and $j$ are indices for molecular species and the source of the continuum absorption, respectively, and $k_{l a}$ and $k_{c a}$ are the line absorption coefficient and the continuum absorption coefficient, respectively.

Fig. 2 shows the path loss caused by the spreading loss and the molecular absorption loss in the THz band as a function of distance and frequency. The parameters used in this figure are as follows: the distance range from $1 \mathrm{~cm}$ to $100 \mathrm{~cm}$, pressure $p=1010 \mathrm{hPa}, \mathrm{RH}=69.6 \%$ and temperature $T=298.55 \mathrm{~K}$. To better show the details of the path loss, values greater than $80 \mathrm{~dB}$ are suppressed to $80 \mathrm{~dB}$. The molecular absorption is occurred at specific frequency so we can see frequency selective fading in LoS path in Fig. 2.

\section{B. Reflected path model}

The power spectrum $\left|H_{m, \text { refl }}\left(f, z_{m}\right)\right|^{2}$ of the reflected path is given by

$$
\left|H_{m, \text { refl }}\left(f, z_{m}\right)\right|^{2}=\left|H_{m, \operatorname{los}}\left(f, z_{m}\right) \cdot R(f)\right|^{2},
$$

where $H_{m, \text { los }}\left(f, z_{m}\right)$ and $R(f)$ are the frequency response of the $m$ th path for the LoS path and the reflection coefficient, respectively. According to Kirchhoff's theory, the reflection coefficient $R(f)$ for a rough surface is given by

$$
R(f)=\gamma(f) \cdot \rho(f),
$$

where $\gamma(f)$ is the smooth surface reflection coefficient from the Fresnel equation for the electromagnetic (EM) wave and $\rho(f)$ is the Rayleigh roughness factor. $\gamma(f)$ is the total Fresnel equation of both of the perpendicular and parallel components $\gamma(f)=\gamma_{\perp}(f)+\gamma_{\|}(f)$. The smooth surface

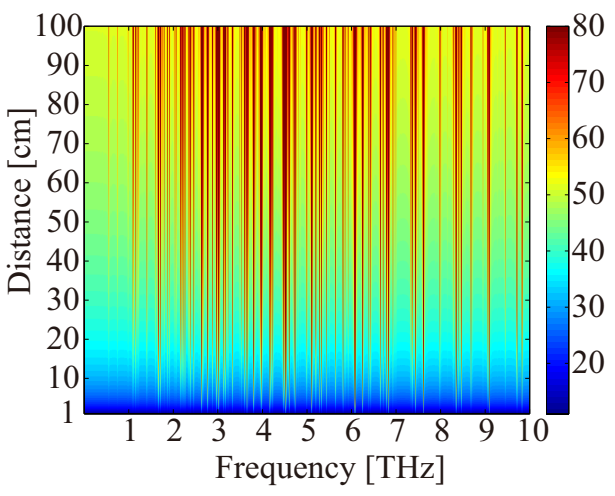

Fig. 2. Path loss due to spreading loss and molecular absorption in the $\mathrm{THz}$ band in the distance range from $1 \mathrm{~cm}$ to $100 \mathrm{~cm}$. Pressure $p=1010 \mathrm{hPa}$, $\mathrm{RH}=69.6 \%$ and temperature $T=298.55 \mathrm{~K}$.

reflection coefficient for the perpendicular component $\gamma_{\perp}(f)$ is described by

$$
\gamma_{\perp}(f)=\frac{n_{i} \cos \theta_{m}-n_{t} \sqrt{1-\left(\frac{n_{i}}{n_{t}} \sin \theta_{m}\right)^{2}}}{n_{i} \cos \theta_{m}+n_{t} \sqrt{1-\left(\frac{n_{i}}{n_{t}} \sin \theta_{m}\right)^{2}}},
$$

where $n_{i}, n_{t}$ and $\theta_{m}$ are the refractive index of the air, the reflector and incident angle of $m$ th path, respectively [10]. Without loss of generality, reflection coefficient for the parallel component $\gamma_{\|}(f)$ can be obtained by a similar approach. The refractive index of the reflector $n_{t}$ is frequency dependent. But so is $n_{i}$, actually. The rough surface effect is characterized by the Rayleigh roughness factor $\rho(f)$ as

$$
\rho(f)=\exp \left(-\frac{8 \pi \cdot f^{2} \cdot \sigma^{2} \cdot \cos ^{2} \theta_{m}}{c^{2}}\right) .
$$

We assume that the height of the rough surface has a Gaussian distribution with standard deviation $\sigma$. This assumption is valid for many indoor building materials [11].

\section{TIME DOMAIN CHANNEL MODEL}

Our methodology obtains the phase component from the transmittance which is amplitude component. Specifically, the method employs the Hilbert transform to obtain the phase component leading to a physically valid impulse response. The received signal $y(t)$ at time $t$ can be obtained by the convolution between the transmitted signal $x(t)$ and the impulse response, $h(\tau, z)$, as

$$
y(t)=\int_{-\infty}^{\infty} h(\tau, z) x(t-\tau) d \tau .
$$

The causal impulse response satisfies

$$
h_{\text {causal }}(\tau, z)= \begin{cases}h_{\text {causal }}(\tau, z) & \tau \geq \tau_{p} \\ 0 & \tau<\tau_{p},\end{cases}
$$


where $\tau_{p}$ is the propagation delay. The frequency response of $h_{\text {causal }}(\tau, z)$ is given by

$$
H(f, z)=e^{-j 2 \pi f \tau_{p}} \int_{0}^{\infty} h_{\text {causal }}\left(\tau+\tau_{p}, z\right) e^{-j 2 \pi f \tau} d \tau .
$$

$H^{\prime}(f, z)$ is defined as causal frequency response in this paper and is defined by

$$
H^{\prime}(f, z)=\int_{0}^{\infty} h_{\text {causal }}\left(\tau+\tau_{p}, z\right) e^{-j 2 \pi f \tau} d \tau,
$$

which leads to $H(f, z)=e^{-j 2 \pi f \tau_{p}} H^{\prime}(f, z)$. Let $\exp [-\alpha(f, z)]$ and $\phi(f, z)$ denote the amplitude and phase components of $H^{\prime}(f, z)$, respectively, i.e., $\exp [-\alpha(f, z)]=$ $\left|H^{\prime}(f, z)\right|=|H(f, z)|$ and $\phi(f, z)=\arg \left(H^{\prime}(f, z)\right)$. Then, $H^{\prime}(f, z)$ for LoS path is given by [8], [12]

$$
H^{\prime}(f, z)=\exp [-\alpha(f, z)+j \phi(f, z)],
$$

and $H^{\prime}(f, z)$ for reflected paths is

$$
H^{\prime}(f, z)=\exp [-\alpha(f, z)+j(\phi(f, z)+\pi)] .
$$

Since the impulse response satisfies causality, $\alpha(f, z)$ and $\phi(f, z)$ are Hilbert transform pairs [8], [12]. Therefore, $\phi(f, z)$ is given by

$$
\phi(f, z)=\frac{1}{\pi} \mathrm{PV} \int_{-\infty}^{\infty} \frac{\alpha\left(f^{\prime}, z\right)}{f-f^{\prime}} d f^{\prime},
$$

where PV represents Cauchy principal value [13]. Given $|H(f, z)|, H(f, z)$ is available based on (13)-(17). Finally, the causal impulse response is given by

$$
h_{\text {causal }}(\tau, z)=\mathcal{F}^{-1}[H(f, z)] .
$$

Finally, frequency band in $\mathrm{THz}$ band for multipath is get by band pass filter (BPF). In BPF scenario, the impulse response $h^{\mathrm{FB}}(\tau)$ can be obtained by

$$
h^{\mathrm{FB}}\left(\tau, f_{c}\right)=\int_{-\infty}^{\infty} h_{\text {causal }}\left(\tau^{\prime}\right) h_{r c}\left(\tau-\tau^{\prime}, B, f_{c}\right) d \tau^{\prime} .
$$

where $B$ is the transmission band, and $f_{c}$ is the center frequency and $h_{r c}\left(\tau, B, f_{c}\right)$ represents the effect of the BPFs. We employ root raised cosine filters as the BPF at both transmitter and receiver. $B$ and $f_{c}$ determine the frequency band.

\section{Characteristic OF THE IMPUlSe ReSPONSE}

In this section, we discuss characteristic of the impulse response in terms of distance, multipath, and frequency band.

First, we focus on the impulse response in the LoS path at $z_{1}=10 \mathrm{~cm}$ and $80 \mathrm{~cm}$ in Figs. 3 and 4 . As increasing distance, the impact of delayed wave is also increasing which come from frequency selective fading in $\mathrm{THz}$ band.

Impulse responses of multipath at $z_{1}=10 \mathrm{~cm}$ and $80 \mathrm{~cm}$ are in Figs. 5 and 6 . The time 0 in these figures means the arrival time of the first wave and the arrival time of the reflected wave is 6.6 ps and 0.8 ps in Figs. 5 and 6, respectively. We can see that first wave at $z_{1}=80 \mathrm{~cm}$ is small than at $z_{1}=10 \mathrm{~cm}$. As

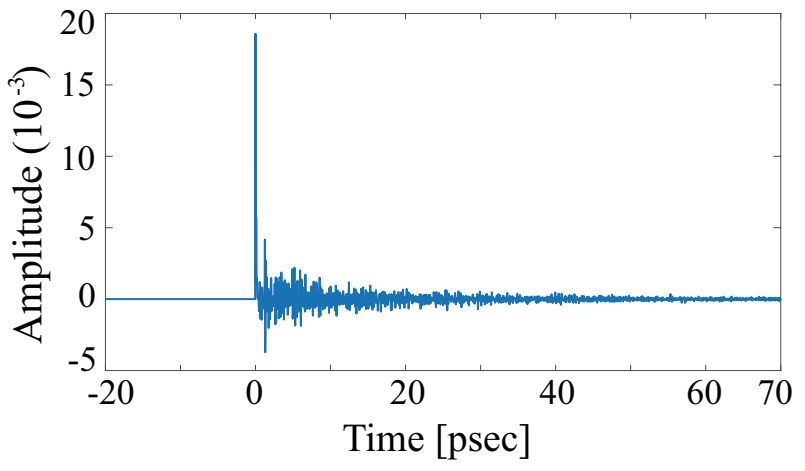

Fig. 3. Impulse response for LoS path in $z_{1}=10 \mathrm{~cm}$. Pressure $p=1010$ $\mathrm{hPa}, \mathrm{RH}=69.6 \%$, and temperature $T=298.55 \mathrm{~K}$.

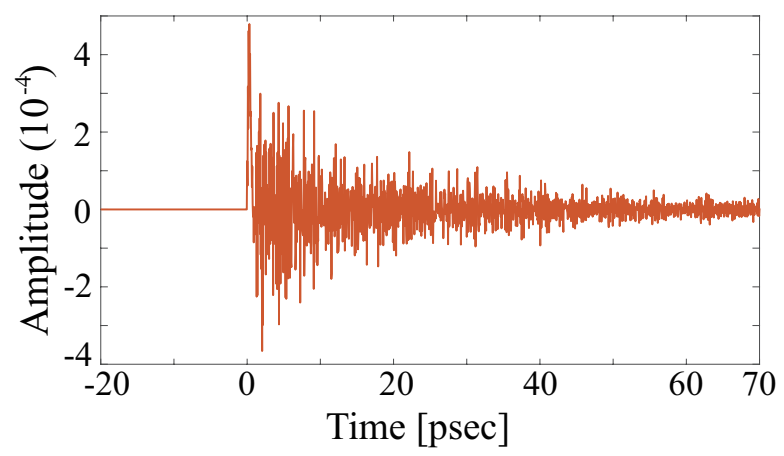

Fig. 4. Impulse response for LoS path in $z_{1}=80 \mathrm{~cm}$. Pressure $p=1010$ $\mathrm{hPa}, \mathrm{RH}=69.6 \%$, and temperature $T=298.55 \mathrm{~K}$.

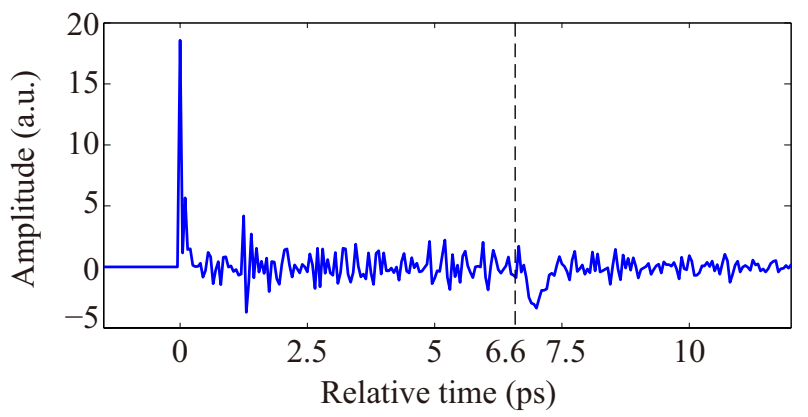

Fig. 5. Impulse response for multipath environment in $z_{1}=10 \mathrm{~cm}$. Pressure $p=1010 \mathrm{hPa}, \mathrm{RH}=69.6 \%$, and temperature $T=298.55 \mathrm{~K}$. (C) 2019 IEEE. Reprinted, with permission, from IEEE Transaction on Terahertz Science and Technology.

$z_{1}$ is increasing, spreading loss and molecular absorption loss in both of LoS path and reflected path are increasing. Still, the impact of reflected wave to LoS path at $z_{1}=80 \mathrm{~cm}$ is stronger than $z_{1}=10 \mathrm{~cm}$, relatively.

In Fig. 7 and 8, the impulse response for frequency band whose bandwidth $B=0.3 \mathrm{THz}$ for multipath at $z_{1}=10 \mathrm{~cm}$ at $f_{c}=0.3 \mathrm{THz}$ and $7.15 \mathrm{THz}$, respectively. (a) in both of figures are the impulse response for LoS path and (b) are the impulse response for multipath. At $0.3 \mathrm{THz}$ the impulse response has strong delayed response than LoS path. However, at $7.15 \mathrm{THz}$, the difference of impulse response between multipath and LoS 


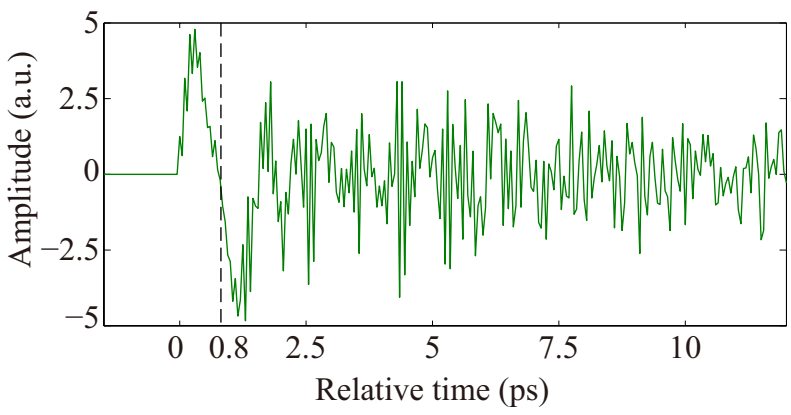

Fig. 6. Impulse response for multipath environment in $z_{1}=80 \mathrm{~cm}$. Pressure $p=1010 \mathrm{hPa}, \mathrm{RH}=69.6 \%$, and temperature $T=298.55 \mathrm{~K}$. (C)2019 IEEE. Reprinted, with permission, from IEEE Transaction on Terahertz Science and Technology.
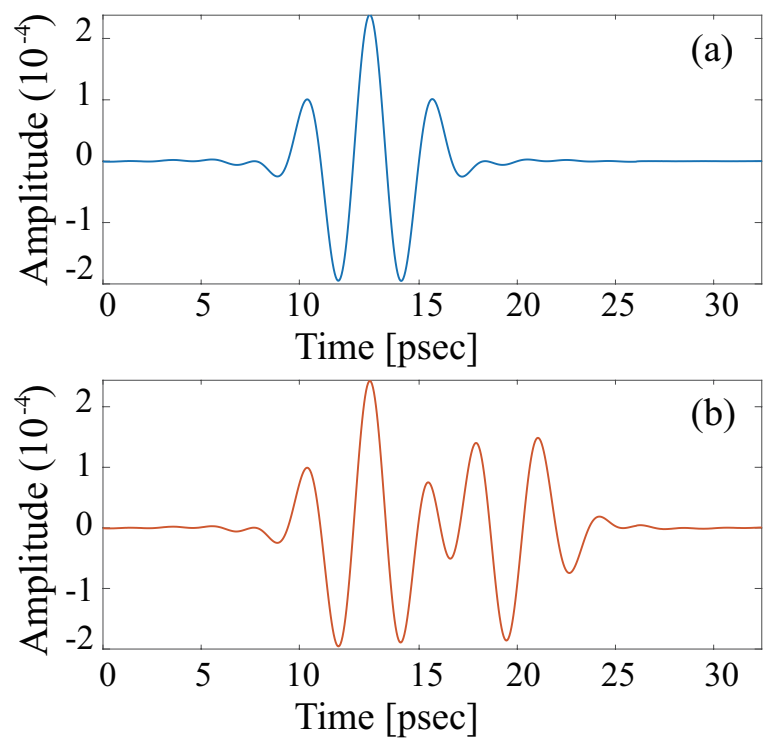

Fig. 7. (a) Impulse response for frequency band $B=0.3 \mathrm{THz}$ for LoS path in $z_{1}=10 \mathrm{~cm}$ at $f_{c}=0.3 \mathrm{THz}$. (b) Impulse response for frequency band $B=0.3 \mathrm{THz}$ for multipath in $z_{1}=10 \mathrm{~cm}$ at $f_{c}=0.3 \mathrm{THz}$. Pressure $p=1010 \mathrm{hPa}, \mathrm{RH}=69.6 \%$, and temperature $T=298.55 \mathrm{~K}$.

path is negligible. It is multipath fading which comes from the reflected wave.

\section{CONCLUSION}

This paper introduced the causal impulse response as a time domain channel model in the $\mathrm{THz}$ band. We explained both of frequency domain and time domain channel model for $\mathrm{THz}$ band. We can see frequency selective fading by molecular absorption in $\mathrm{THz}$ band in LoS. At $z_{1}=80 \mathrm{~cm}$, reflected path has remarkable effect to LoS path. Additionally, frequency band results show us necessary to select proper band for $\mathrm{THz}$ communication.

\section{ACKNOWLEDGMENT}

The work of Joonas Kokkoniemi and Janne Lehtomäki under TERRANOVA project has received funding from Horizon 2020, European Union's Framework Programme for Research and Innovation, under grant agreement No. 761794.
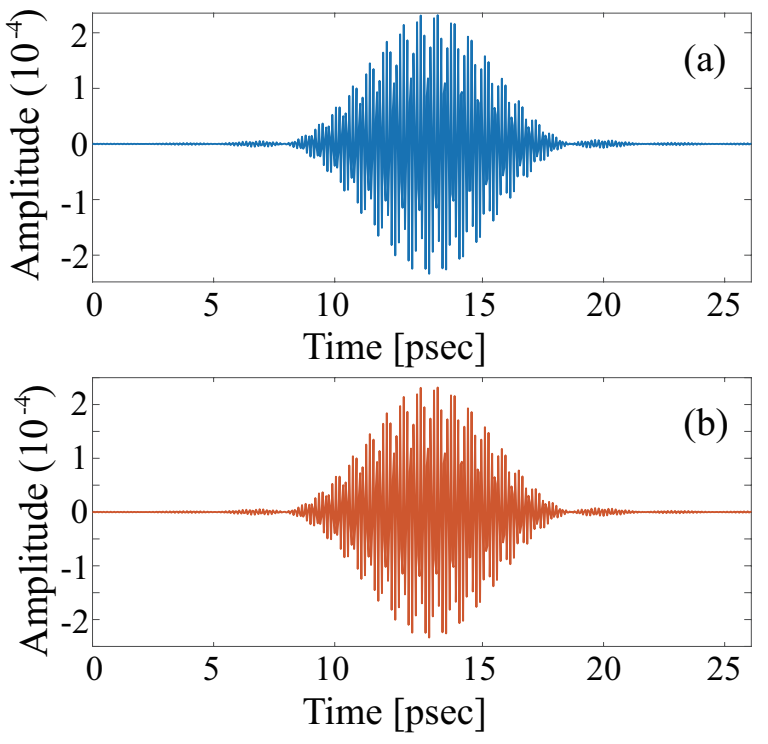

Fig. 8. (a) Impulse response for frequency band $B=0.3 \mathrm{THz}$ for LoS path in $z_{1}=10 \mathrm{~cm}$ at $f_{c}=7.15 \mathrm{THz}$. (b) Impulse response for frequency band $B=0.3 \mathrm{THz}$ for multipath in $z_{1}=10 \mathrm{~cm}$ at $f_{c}=7.15 \mathrm{THz}$. Pressure $p=1010 \mathrm{hPa}, \mathrm{RH}=69.6 \%$, and temperature $T=298.55 \mathrm{~K}$.

\section{REFERENCES}

[1] I. F. Akyildiz and J. M. Jornet, "Electromagnetic wireless nanosensornetworks," Nano Communication Networks, vol. 1, no. 1, pp. 3-19, Mar 2010.

[2] I. F. Akyildiz, J. M. Jornet, and C. Han, "Terahertz band: Next frontier for wireless communications," Physical Communication, vol. 12, pp. 16-32, Sep 2014.

[3] J. M. Jornet and I. F. Akyildiz, "Channel modeling and capacity analysis for electromagnetic wireless nanonetworks in the terahertz band," IEEE Transactions on Wireless Communications, vol. 10, no. 10, pp. 32113221, Oct 2011

[4] J. Kokkoniemi, J. Lehtomäki, K. Umebayashi, and M. Juntti, "Frequency and time domain channel models for nanonetworks in terahertz band," IEEE Transactions on Antennas and Propagation, vol. 63, no. 2, pp. 678-691, Feb 2015.

[5] J. M. Jornet and I. F. Akyildiz, "Femtosecond-long pulse-based modulation for terahertz band communication in nanonetworks," IEEE Transactions on Communications, vol. 62, no. 5, pp. 1742-1754, May 2014.

[6] K. Tsujimura, K. Umebayashi, J. Kokkoniemi, J. Lehtomaki, and Y. Suzuki, "A causal channel model for thz band," IEEE Transaction on THz science and technology, vol. 8, no. 1, pp. 52-62, Jan. 2018.

[7] C. Han, A. O. Bicen, and I. F. Akyildiz, "Multi ray channel modeling and wideband characterization for wireless communications in the terahertz band," IEEE Transactions on Wireless Communications, vol. 14, no. 5, pp. 2402-2412, May 2015.

[8] S. Paine, "The am atmospheric model," Smithsonian Astrophysical Observatory, SMA Technical Memo 152, Feb 2012.

[9] L. S. Rothman, I. E. Gordon, A. Barbe et al., "The HITRAN 2008 molecular spectroscopic database," Journal of Quantitative Spectroscopy and Radiative Transfer, vol. 110, no. 9-10, pp. 533-572, Jun-July 2009.

[10] S. J. Adams, Electromagnetic theory. McGraw-Hill BookCompany, 1941.

[11] C. Jansen et al., "Diffuse scattering from rough surfaces in thz communication channels," IEEE Transactions on Terahertz Science and Technology, vol. 1, no. 2, pp. 462-472, Nov. 2011.

[12] H. Kuzmany, Solid-State Spectroscopy. Springer, 1998.

[13] I. S. Gradshteyn and I. M. Ryzhik, Tables of integrals Series and Products, 7th ed. Academic Press, 1992.

[14] B. Sklar, Digital Communications: Fundamentals and Applications, 2nd ed. Prentice Hall, 2001. 